\title{
A Chinese Herbal Decoction, Huoxue Qingyi Decoction, Promotes Rehabilitation of Patients with Severe Acute Pancreatitis: A Retrospective Study
}

\author{
Chao Hui Ji, ${ }^{1}$ Cheng Wu Tang, ${ }^{2}$ Wen Ming Feng, ${ }^{2}$ Ying Bao, ${ }^{2}$ and Li Qin Yao ${ }^{2}$ \\ ${ }^{1}$ Department of Emergency Intensive Care Unit, First People's Hospital Affiliated to Huzhou University Medical College, \\ Huzhou, Zhejiang 313000, China \\ ${ }^{2}$ Department of General Surgery, First People’s Hospital Affiliated to Huzhou University Medical College, Huzhou, \\ Zhejiang 313000, China
}

Correspondence should be addressed to Li Qin Yao; 47180177@qq.com

Received 10 January 2016; Revised 8 March 2016; Accepted 13 March 2016

Academic Editor: Chang-Gue Son

Copyright (c) 2016 Chao Hui Ji et al. This is an open access article distributed under the Creative Commons Attribution License, which permits unrestricted use, distribution, and reproduction in any medium, provided the original work is properly cited.

\begin{abstract}
Severe acute pancreatitis (SAP) still remains an important surgical problem with high morbidity and mortality. The utilization of Traditional Chinese Medicine shows good prospects in therapy of SAP since it has advantages of more extensive pharmacological effects and fewer adverse effects. In this retrospective study, 38 patients received standardized treatment (control group) and 37 patients received Chinese herbal decoction, Huoxue Qingyi Decoction (HQD group), in addition to standard treatment for SAP. We found that the HQD group had a shorter hospital stay and lower initial expense than the control group $(P<0.05)$. The duration of hyperamylasemia and systemic inflammatory response syndrome (SIRS) were significantly shorter in HQD group $(P<0.05)$. The percentage of patients having any complication was much lower in HQD group than control group $(27 / 38$ versus $17 / 37, P<0.05)$, especially pancreatic pseudocyst $(10 / 38$ versus $2 / 37, P<0.05)$. No adverse effect induced by HQD was found. We concluded that the HQD was effective, safe, and economic for reduction of complication, for early recovery from systemic inflammation, and for promoting earlier rehabilitation from SAP.
\end{abstract}

\section{Introduction}

Severe acute pancreatitis (SAP) is a common cause of emergency hospital admission, with an increase in the incidence rate during the past 30 years. It can induce vascular leakage, shock, systemic inflammatory response syndrome, and even organ dysfunctions $[1,2]$. Although many efforts have been contributed to the research in the disease, SAP is still dangerous and has many complications accompanied by a high mortality rate. Therefore, studies on improvement in therapy of SAP are urgently needed.

In contrast to western medicine, there is accumulating evidence suggestive of beneficial effects of plants used in Traditional Chinese Medicine (TCM) and compounds isolated from medicinal plants $[3,4]$. The utilization of TCM shows good prospects in therapy of SAP since it has advantages of more extensive pharmacological effects and fewer adverse effects $[5,6]$. The aim of the present retrospective study was to clarify the validity of the Chinese Herbal Decoction, Huoxue Qingyi Decoction (HQD), in patients with SAP.

\section{Methodology}

2.1. Patients. Between June 2010 and June 2014, 75 patients with a clinical diagnosis of SAP of whom 49 were men and 26 were women with a mean age of 45 years (range: 22 to 69 years) were admitted to the Department of Emergency Intensive Care Unit at First People's Hospital affiliated to Huzhou University Medical College (Zhejiang Province, China) within 3 days of disease onset. 38 patients received standardized medical therapy for SAP (control group) and 37 patients received additional HQD therapy (HQD group). Medical records were retrospectively reviewed. 
TABle 1: Components of Huoxue Qingyi Decoction with Latin and English names.

\begin{tabular}{llc}
\hline Name in Latin & Name in English & Dose \\
\hline Salviae Miltiorrhizae & Danshen root & $30 \mathrm{~g}$ \\
Desmodium styracifolium & Snowbell leaf tick clover herb & $30 \mathrm{~g}$ \\
Rheum officinale Baill & Rhubarb & $10 \mathrm{~g}$ \\
Natrii Sulfas & Glauber salt & $9 \mathrm{~g}$ \\
Magnolia officinalis & Magnolia bark & $10 \mathrm{~g}$ \\
Radix Paeoniae Alba & White peony root & $10 \mathrm{~g}$ \\
Radix Bupleuri & Bupleurum root & $10 \mathrm{~g}$ \\
Fructus Aurantii & Immature bitter orange & $10 \mathrm{~g}$ \\
Immaturus & Scutellaria root & $10 \mathrm{~g}$ \\
Scutellaria baicalensis & & \\
\hline
\end{tabular}

The diagnostic and classification criteria for SAP were in accordance with the revised Atlanta criteria of 2013 [7], including two of the following features: (1) abdominal pain consistent with AP; (2) amylase activity at least three times greater than the upper limit of normal; and (3) characteristic findings of AP on contrast-enhanced computed tomography (CT) and, less commonly, on magnetic resonance imaging or transabdominal ultrasonography. Patients who had cardiac failure or pulmonary edema, were pregnant, underwent early surgical treatment or other TCM prior to hospital admission, or failed to give consent were excluded.

This study was conducted in accordance with the principles of the Declaration of Helsinki and "Good Clinical Practice" guidelines. Written informed consent was obtained from all patients. Approval of the study was obtained from the institutional review board.

2.2. Treatment. All of the patients received standardized medical therapy for SAP according to the United Kingdom, Chinese Medical Association, and International Association of Pancreatology guidelines [8-10]. In addition, patients in the HQD group received a $200 \mathrm{~mL}$ enema of HQD every 12 hours for 8 days and $50 \mathrm{~mL}$ of the same decoction either intragastrically or orally every 8 hours for 8 days.

The composition of HQD (shown in Table 1) consisted of Salviae miltiorrhizae $30 \mathrm{~g}$, Desmodium styracifolium $30 \mathrm{~g}$, Rheum officinale Baill $10 \mathrm{~g}$, Natrii Sulfas $9 \mathrm{~g}$, Magnolia officinalis 10 g, Radix Paeoniae Alba 10 g, Radix Bupleuri 10 g, Fructus Aurantii Immaturus $10 \mathrm{~g}$, and Scutellaria baicalensis $10 \mathrm{~g}$, which were purchased from Hangzhou $\mathrm{Hu}$ Qing Yu Tang Pharmaceutical Co., Ltd. (Hangzhou, China), whose products meet the commercial quality control according to the China Pharmacopoeia 2010. Herbal mixtures were made based on remedy menu by an experienced Chinese medicine practitioner.

2.3. Outcome Measurements. Clinical factors such as gender, age, body mass index (BMI), body weight, underlying comorbidity, etiological factor, severity of pancreatitis (Ranson's signs and Balthazar CT index), operability, hyperamylasemia duration, mechanical ventilation, mortality rate, hospital stay, initial hospitalization cost, and adverse effects were compared. All complications such as acute respiratory distress syndrome (ARDS), renal failure, hemorrhage, sepsis, pancreatic pseudocyst, and pancreatic abscess from admission to discharge from hospital were retrospectively analyzed. Respiratory failure was defined as the requirement for mechanical ventilation beyond 24 hours after surgery. ARDS and multiple organ failure were defined as per Bone et al. [11]. Sepsis required evidence of systemic inflammatory response syndrome (SIRS) with microbiological evidence of infection. SIRS was diagnosed by clinical manifestation of 2 or more of the following features: systolic blood pressure $<90 \mathrm{mmHg}$, tachycardia $>90 / \mathrm{min}$, respiratory rate $>20 /$ min or peripheral arterial $\mathrm{CO}_{2}$ tension $\left(\mathrm{PaCO}_{2}\right)<32 \mathrm{mmHg}$, temperature $>$ $38.0^{\circ} \mathrm{C}$ or $<36.0^{\circ} \mathrm{C}$, leukocytosis $>12,000 / \mu \mathrm{L}$ or leukopenia $<$ $4000 / \mu \mathrm{L}$, or $10 \%$ immature (band) forms.

2.4. Statistical Analysis. All measurements were expressed as mean $\pm \mathrm{SD}$. The statistical analyses were performed using the two-sample $t$-test and adjusted Chi-square test for the two groups. The exact Chi-square test was also used if individual cell size was less than 5 counts. $P$ value $<0.05$ was considered statistically significant.

\section{Results}

3.1. Patient Characteristics. There were no statistically significant differences between two groups in patient characteristics, in terms of gender, age, body mass index (BMI), severity of pancreatitis (Ranson's signs and Balthazar CT index), body weight, underlying comorbidity, and etiological factor (Table 2).

3.2. Clinical Outcomes. Clinical outcomes were shown in Table 3. The HQD group seemed to have had a shorter hospital stay and lower initial expense than the control group $(P<0.05)$. The duration of hyperamylasemia and SIRS was significantly shorter in HQD group $(P<0.05)$.

The percentage of patients having any complication was much higher in control group than HQD group (27/38 versus $17 / 37, P<0.05$ ) (Table 4 ), but there was no significant difference in in-hospital morbidity between two groups (Table 3 ). Further analysis about complications was performed and pancreatic pseudocyst was found significantly more frequent in control group compared with HQD group $(P<0.05)$ (Table 4). No adverse effect induced by HQD was found.

\section{Discussion}

Severe acute pancreatitis (SAP), a life-threatening condition characterized by edema, inflammation, hemorrhage, and necrosis of the pancreas, still remains an important surgical problem with high morbidity and mortality $[12,13]$. Traditional Chinese Medicine active ingredients, such as emodin, magnolol, naringin, ginkgolide $\mathrm{B}$, sanchinoside, taxol, resveratrol, rutoside, tetramethylpyrazine, and breviscapine, being used in treating SAP have advantages in not only acting on the pancreas, stomach, and intestines, but also having 
TABLe 2: Patient characteristics.

\begin{tabular}{|c|c|c|c|}
\hline & Control group $(n=38)$ & HQD group $(n=37)$ & $P$ value \\
\hline Gender & & & 0.7438 \\
\hline Male & 26 & 23 & \\
\hline Female & 12 & 14 & \\
\hline \multicolumn{4}{|l|}{ Underlying comorbidity } \\
\hline Hypertension & 11 & 12 & 0.9388 \\
\hline Heart disease & 3 & 2 & 0.9754 \\
\hline Diabetes mellitus & 9 & 7 & 0.8245 \\
\hline Fatty liver & 6 & 7 & 0.9578 \\
\hline Chronic obstructive pulmonary disease & 3 & 4 & 0.9704 \\
\hline Etiological factor & & & 0.8336 \\
\hline Biliary tract stone & 17 & 20 & \\
\hline Hyperlipidemia & 9 & 7 & \\
\hline Alcohol intake & 10 & 9 & \\
\hline Other & 2 & 1 & \\
\hline Age (year) & $60.3 \pm 7.1$ & $61.8 \pm 7.2$ & 0.3667 \\
\hline Ranson's signs (score in $48 \mathrm{~h}$ ) & $4.9 \pm 1.2$ & $5.1 \pm 1.1$ & 0.4546 \\
\hline CT index (score in $48 \mathrm{~h}$ ) & $8.7 \pm 2.1$ & $7.9 \pm 2.2$ & 0.1115 \\
\hline $\operatorname{BMI}\left(\mathrm{kg} / \mathrm{m}^{2}\right)$ & $22.7 \pm 3.3$ & $23.2 \pm 3.5$ & 0.5263 \\
\hline Body weight (kg) & $77.3 \pm 9.4$ & $76.2 \pm 8.7$ & 0.6008 \\
\hline
\end{tabular}

TABLE 3: Clinical outcomes.

\begin{tabular}{lccc}
\hline & Control group $(n=38)$ & HQD group $(n=37)$ & $P$ value \\
\hline Hyperamylasemia duration (day) & $5.3 \pm 2.1$ & $3.7 \pm 1.4$ & 0.0001 \\
Hospital stay (day) & $16.1 \pm 4.2$ & $13.4 \pm 5.7$ & 0.0220 \\
Mechanical ventilation & 4 & 1 & 0.6953 \\
Operability & 2 & 0 & 0.9812 \\
In-hospital mortality & 1 & $4.5 \pm 1.7$ & 0.9893 \\
SIRS duration (day) & $6.4 \pm 2.2$ & $27446.7 \pm 1375.6$ & 0.0004 \\
Initial hospitalization cost (Chinese yuan) & $34557.4 \pm 1134.7$ & 0.0000 \\
\hline
\end{tabular}

TABle 4: Complications.

\begin{tabular}{lccr}
\hline & Control group $(n=38)$ & HQD group $(n=37)$ & $P$ value \\
\hline Complication (\%) & $27(71.1 \%)$ & $17(45.9 \%)$ & 1 \\
Hemorrhage & 3 & 2 & 0.0485 \\
Pancreatic pseudocyst & 10 & 2 & 0.6266 \\
Pancreatic abscess & 3 & 4 & 0.0312 \\
Renal failure & 7 & 4 & 0.9514 \\
Heart failure & 4 & 8 & 0.5452 \\
Sepsis & 12 & 5 & 0.9254 \\
Acute respiratory distress syndrome & 8 & 0.5773 \\
\hline
\end{tabular}

marked treatment effects on other visceral injuries arising from systemic inflammatory responses accompanying pancreatitis and blocking the disease progression [14].

Evidences in basic and clinical research suggest that it not only is an injury caused by the activated pancreatic enzymes but also involves pancreatic ischemia which leads to disturbance of pancreatic microcirculation that plays an important role in its pathophysiological processes [15]. The specific local microcirculatory changes cannot be prevented merely by adequate fluid therapy [16]. Salviae Miltiorrhizae, a traditional herbal medicine, has a low price and a wide range of clinical applications. It is proved to be effective in improvement of microcirculatory disturbances, elimination of oxygen free radicals, modulation of the metabolism of lipid inflammatory mediator, and blocking of calcium inflow and prevention of calcium overload [17]. Therefore, HQD in our study was a combination of Salviae Miltiorrhizae and Qingyi Decoction. 
In this study, we found the patients of the HQD group showed a significantly shorter hospital stay, lower initial hospitalization cost, and shorter duration of SIRS and hyperamylasemia than control group. Though there was no significant difference in in-hospital morbidity between two groups, fewer complications especially pancreatic pseudocyst were found in HQD group than in control group. Notably, hemorrhage was not significantly alleviated in spite of antithrombotic properties of Salviae Miltiorrhizae.

Although Chinese herbal medicine is now widely used throughout the world, some herbal medicines have been associated with adverse effects and toxic effects [18]. Among the most serious adverse effects that can be caused by herbal medicine is drug induced liver injury (DILI). However, it was reported that many cases of DILI were induced by the self-purchase and administration of herb medicine, without a doctor's prescription [19]. Another study in Singapore found that $52.95 \%$ (9 of 17) of DILI cases were related to adulterated western medicine in herbal medicine [20]. Moreover, one study in China reported that only $24.2 \%$ of cases were related to herbal medicine and the other $75.8 \%$ to prescribed western medicine [21]. The current study showed that HQD did not induce any adverse effects including DILI.

In conclusion, HQD administrated per rectum and intragastrically or orally was well tolerable and did not increase the frequency of hemorrhage or adverse effects, at least in the short term. Therefore, application of HQD was effective, safe, and economic for reduction of complication, for early recovery from systemic inflammation, and for promoting earlier rehabilitation from SAP, though the precise mechanisms of therapeutic effects of $\mathrm{HQD}$ on patients required further exploration. Because of the retrospective nature and the small sample size, further prospective study with large sample size is needed to confirm the results of our study.

\section{Competing Interests}

The authors declare that they have no conflict of interests.

\section{Authors' Contributions}

Chao Hui Ji and Li Qin Yao designed the study and wrote the paper; Wen Ming Feng and Cheng Wu Tang conducted patients' management and monitoring; Ying Bao provided the collection of all of the human material in addition to providing financial support for this work.

\section{References}

[1] B. M. Rau, A. Bothe, M. Kron, and H. G. Beger, "Role of early multisystem organ failure as major risk factor for pancreatic infections and death in severe acute pancreatitis," Clinical Gastroenterology and Hepatology, vol. 4, no. 8, pp. 1053-1061, 2006.

[2] R. Talukdar and S. S. Vege, "Acute pancreatitis," Current Opinion in Gastroenterology, vol. 31, no. 5, pp. 374-379, 2015.

[3] R.-L. Ji, S.-H. Xia, Y. Di, and W. Xu, "Mechanism and doseeffect of Ginkgolide B on severe acute pancreatitis of rats," World Journal of Gastroenterology, vol. 17, no. 17, pp. 2241-2247, 2011.
[4] W.-G. Yu, G. Xu, G.-J. Ren et al., "Preventive action of curcumin in experimental acute pancreatitis in mouse," Indian Journal of Medical Research, vol. 134, no. 11, pp. 717-724, 2011.

[5] Y.-Y. Li, A. Sibaev, M.-Z. Zhou, G.-Y. Zhu, B. Yüce, and M. Storr, "The Chinese herbal preparation Qing Yi Tang (QYT) improves intestinal myoelectrical activity and increases intestinal transit during acute pancreatitis in rodents," Phytotherapy Research, vol. 21, no. 4, pp. 324-331, 2007.

[6] J.-W. Zhang, G.-X. Zhang, H.-L. Chen et al., “Therapeutic effect of Qingyi decoction in severe acute pancreatitis-induced intestinal barrier injury," World Journal of Gastroenterology, vol. 21, no. 12, pp. 3537-3546, 2015.

[7] P. A. Banks, T. L. Bollen, C. Dervenis et al., "Classification of acute pancreatitis-2012: revision of the Atlanta classification and definitions by international consensus," Gut, vol. 62, no. 1, pp. 102-111, 2012.

[8] Group of Pancreas Surgery, Chinese Society of Surgery, and Chinese Medical Association, "The guideline of diagnosis and treatment of severe acute pancreatitis," Zhonghua Wai Ke Za Zhi, vol. 45, no. 11, pp. 727-729, 2007.

[9] W. Uhl, A. Warshaw, C. Imrie et al., "IAP guidelines for the surgical management of acute pancreatitis," Pancreatology, vol. 2, no. 6, pp. 565-573, 2002.

[10] Working Party of the British Society of Gastroenterology, Association of Surgeons of Great Britain and Ireland, Pancreatic Society of Great Britain and Ireland, and Association of Upper GI Surgeons of Great Britain and Ireland, "UK guidelines for the management of acute pancreatitis," Gut, vol. 54, supplement 3, pp. 1-9, 2005.

[11] R. C. Bone, W. J. Sibbald, and C. L. Sprung, "The ACCP-SCCM consensus conference on sepsis and organ failure," Chest, vol. 101, no. 6, pp. 1481-1483, 1992.

[12] H. K. Hu, X. J. Du, A. Li, N. W. Ke, and W. M. Hu, "A minimally invasive management for abdominal compartment syndrome in severe acute pancreatitis," Pakistan Journal of Medical Sciences, vol. 29, no. 1, pp. 231-233, 2012.

[13] A. T. Ince and B. Baysal, "Pathophysiology, classification and available guidelines of acute pancreatitis," Turkish Journal of Gastroenterology, vol. 25, no. 4, pp. 351-357, 2014.

[14] W. Chen, X. Yang, L. Huang et al., "Qing-Yi decoction in participants with severe acute pancreatitis: a randomized controlled trial," Chinese Medicine, vol. 10, article 11, 2015.

[15] C. M. Cuthbertson and C. Christophi, "Disturbances of the microcirculation in acute pancreatitis," British Journal of Surgery, vol. 93, no. 5, pp. 518-530, 2006.

[16] J. Panek, J. Zasada, and M. Poźniczek, "Microcirculatory disturbance in the course of acute pancreatitis," Przeglad Lekarski, vol. 64, no. 6, pp. 435-437, 2007.

[17] Z. Xiping, W. Dijiong, L. Jianfeng et al., "Effects of salvia miltiorrhizae on ICAM-1, TLR4, NF- $\kappa$ B and bax proteins expression in multiple organs of rats with severe acute pancreatitis or obstructive jaundice," Inflammation, vol. 32, no. 4, pp. 218-232, 2009.

[18] C. Calitz, L. du Plessis, C. Gouws et al., "Herbal hepatotoxicity: current status, examples, and challenges," Expert Opinion on Drug Metabolism and Toxicology, vol. 11, no. 10, pp. 1551-1565, 2015.

[19] S. W. Nam, J. T. Baek, D. S. Lee, S. B. Kang, B. M. Ahn, and K. W. Chung, "A case of acute cholestatic hepatitis associated with the seeds of Psoralea corylifolia (Boh-Gol-Zhee)," Clinical Toxicology, vol. 43, no. 6, pp. 589-591, 2005. 
[20] C.-T. Wai, B.-H. Tan, C.-L. Chan et al., "Drug-induced liver injury at an Asian center: a prospective study," Liver International, vol. 27, no. 4, pp. 465-474, 2007.

[21] B. Li, Z. Wang, J.-J. Fang, C.-Y. Xu, and W.-X. Chen, "Evaluation of prognostic markers in severe drug-induced liver disease," World Journal of Gastroenterology, vol. 13, no. 4, pp. 628-632, 2007. 


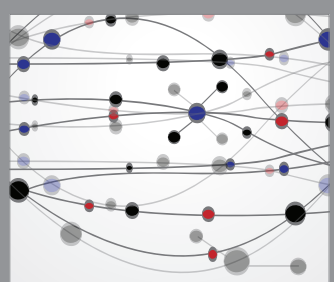

The Scientific World Journal
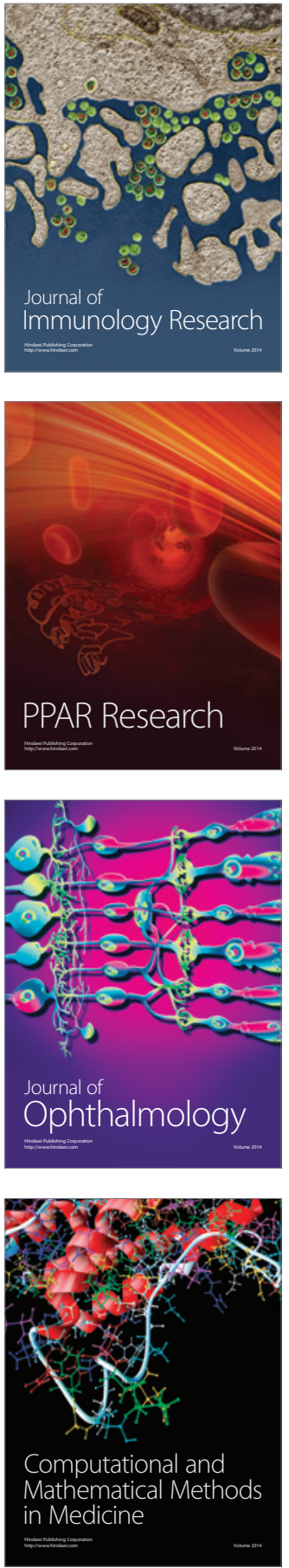

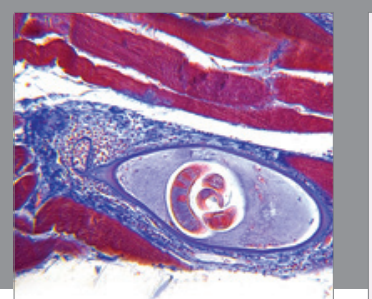

Gastroenterology Research and Practice

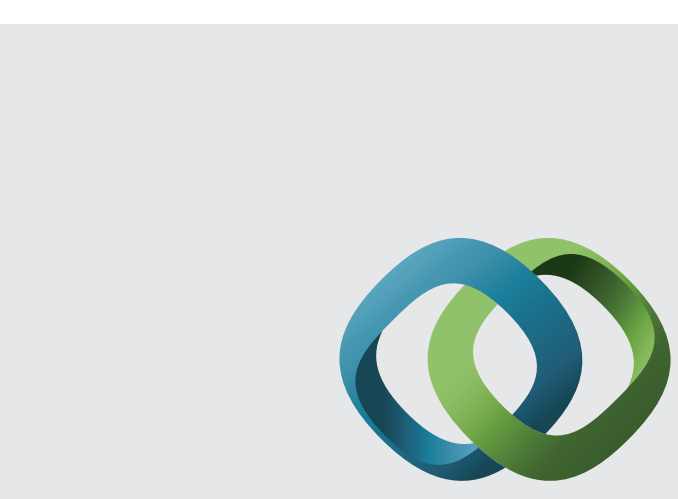

\section{Hindawi}

Submit your manuscripts at

http://www.hindawi.com
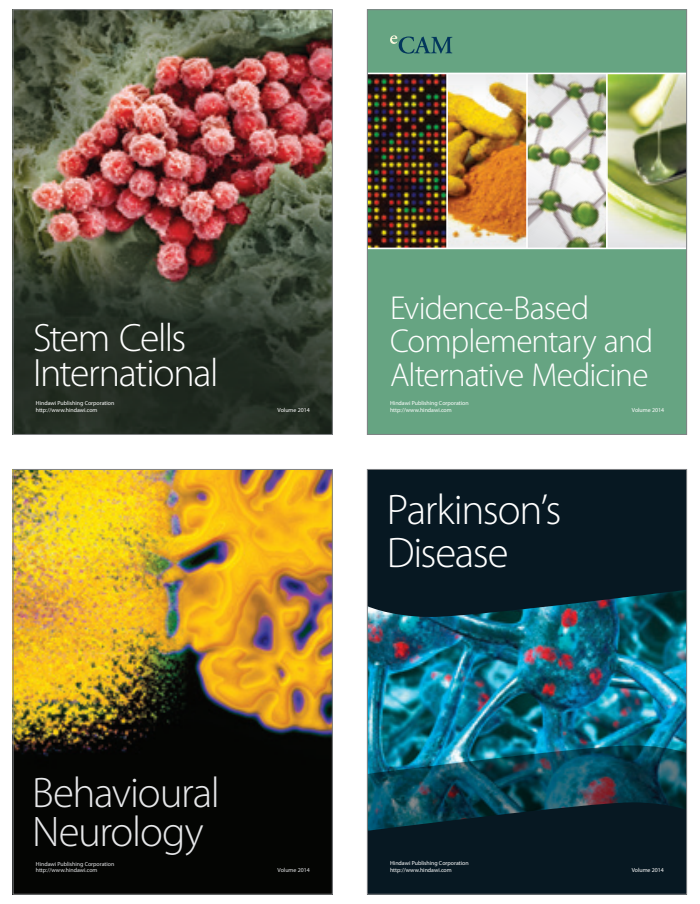
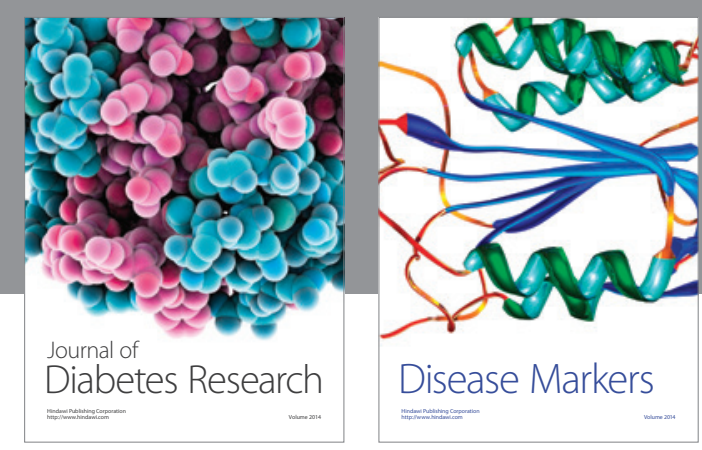

Disease Markers
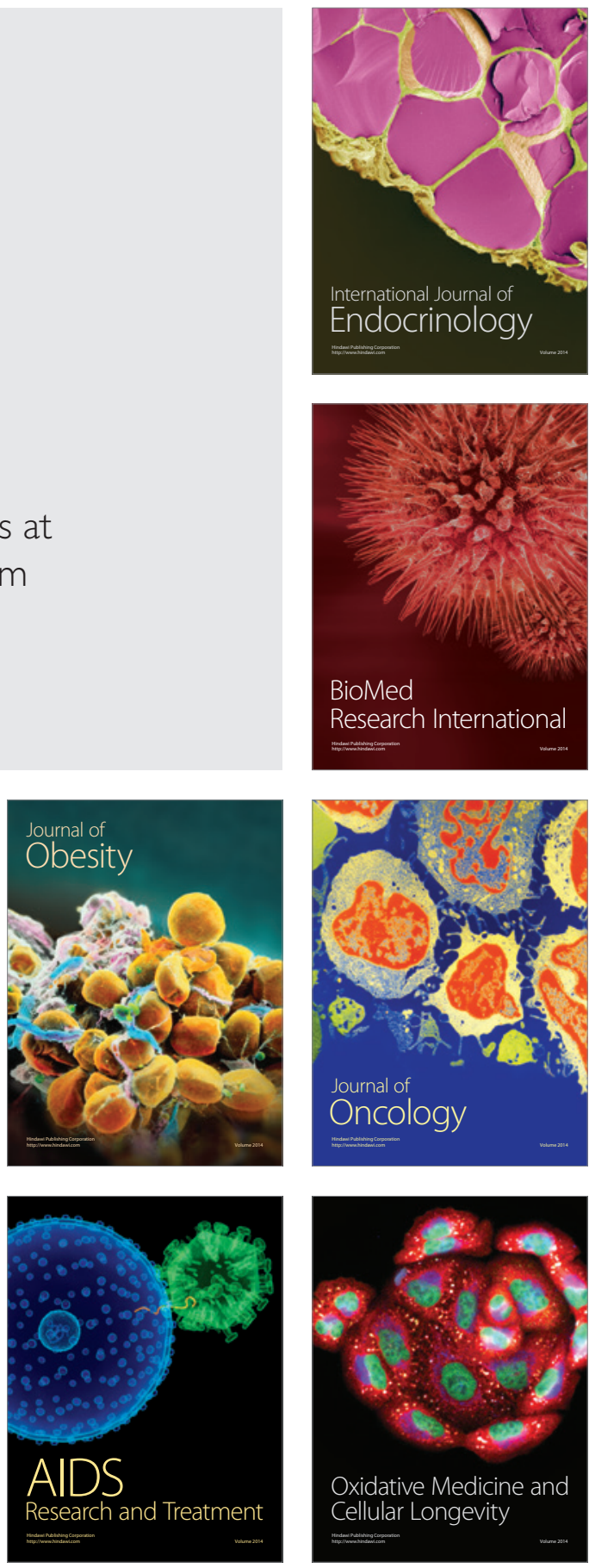